\title{
Luminosities, Space Densities and Redshift Distributions of Gamma-Ray Bursts
}

\author{
Maarten Schmidt \\ California Institute of Technology, Pasadena, California 91125
}

\begin{abstract}
We use the BD2 sample of gamma-ray bursts (GRBs) based on 5.9 years of BATSE DISCLA data with a variety of models of the luminosity function to derive characteristic GRB luminosities, space densities and redshift distributions. Previously published results for an open universe and modest density evolution of the GRBs showed characteristic peak luminosities around $5 \times 10^{51} \mathrm{ergs} \mathrm{s}^{-1}$ in the $50-300 \mathrm{keV}$ band if the emission is isotropic, and local space densities around $0.2 \mathrm{Gpc}^{-3} \mathrm{y}^{-1}$. In this paper, we illustrate for several luminosity function models the predicted distributions of peak flux, luminosity and redshifts. We use the luminosity function models also to address the connection between supernovae and GRB. If all supernovae of type $\mathrm{Ib} / \mathrm{c}$ harbor a GRB, the beaming fraction would have to be in the range $10^{-5}-10^{-3.5}$. We find that GRB 980425, if correctly identificd with SN 1998bw, has to be part of a population different from that of the bulk of GRBs.
\end{abstract}

\section{INTRODUCTION}

The luminosity function and cosmological evolution of extragalactic objects are usually derived from observed samples that are complete above a given flux limit and have measured redshifts. In the case of gamma-ray bursts (GRBs), there is no well defined complete sample with redshifts available at the present time. Under these circumstances, we have found it useful to invert the process, to assume a luminosity function, and to derive intrinsic properties of GRBs such as their characteristic peak luminosity $L^{*}$ and local space density [9]. In this paper, we illustrate the predictions for some of these luminosity function models. We also use these models to address the issue of a connection between supernovae and GRBs.

\section{MODELS OF THE GRB LUMINOSITY FUNCTION}

We start with a brief description of the methodology. For a detailed description of the models, the reader is referred to [9]. We used luminosity functions that were broken power laws with characteristic luminosity $L^{*}$, with different slopes and

CP526, Gamma-Ray Bursts: $5^{\text {th }}$ Huntsville Symposium, edited by R. M. Kippen, et al. () 2000 American Institute of Physics 1-56396-947-5/00/\$17.00 

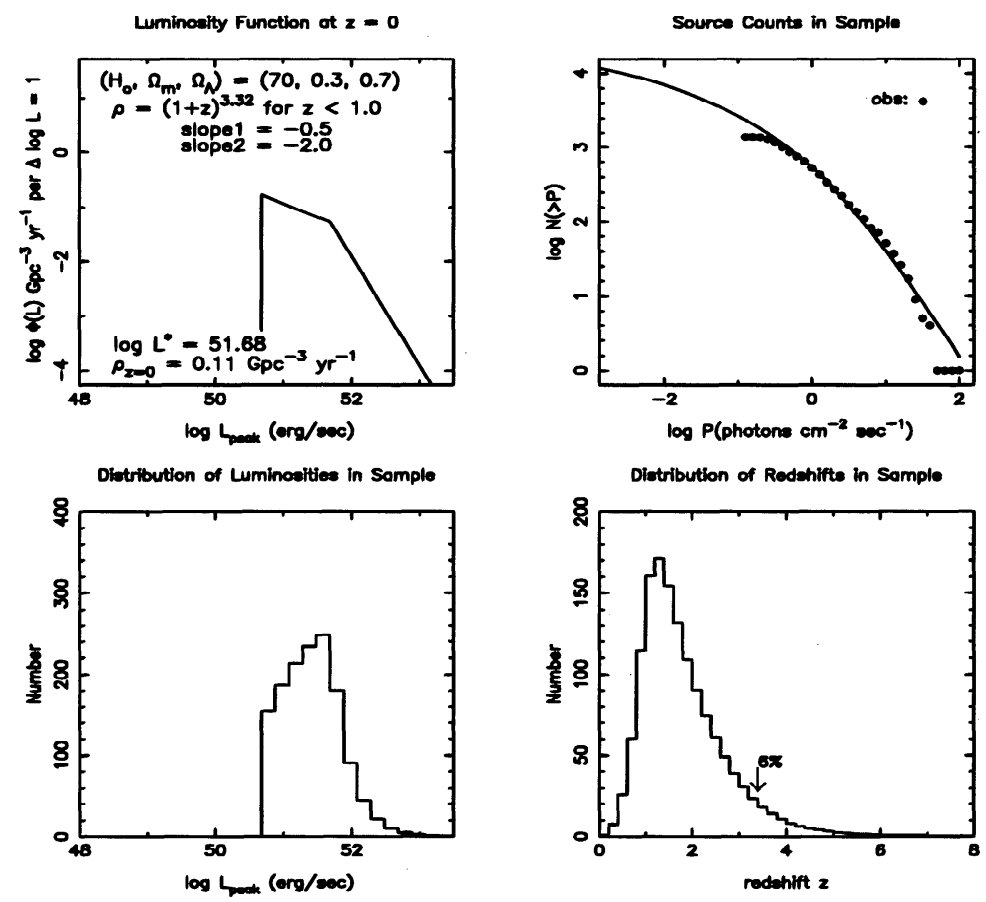

FIGURE 1. Assuming a luminosity function extending from $L^{*} / 10$ to $100 L^{*}$ characterized in the upper left panel and the text, the other panels show the predicted luminosity distribution, source counts and redshift distributions for the BD2 sample of GRBs

different extents above and below $L^{*}$. In addition, we assumed density evolution rising to a factor of 10 at $z=1$ and constant for $z>1$. We used the BD2 sample of 1391 GRB which was derived from 5.9 years of BATSE DISCLA data on a timescalc of $1024 \mathrm{~ms}[9,10]$. For a given luminosity function model, the observed euclidean value of $V / V_{\max }=0.334 \pm 0.008$ allowed a robust determination of $L^{*}$. The total number of GRBs in the BD2 sample provided the normalization of the luminosity function. For a given cosmology and evolution, the value of $L^{*}$ ranged over a factor of 6 and the local space density over a factor of 2 . Typical values ${ }^{1}$ for an open universe with $q_{o}=0.1$ were a characteristic peak luminosity $L^{*}=5 \times 10^{51}$ ergs $\mathrm{s}^{-1}$ and a local space density around $0.2 \mathrm{Gpc}^{-3} \mathrm{yr}^{-1}$. Beaming reduces the peak luminosity and increases the density by the same factor.

We illustrate in this paper the results based on several different luminosity functions. We are using a cosmological model that is a flat accelerating universe with a matter density $\Omega_{m}=0.3$ and cosmological constant $\Omega_{\Lambda}=0.7$ [1]. We assume a Band type GRB spectrum [2] with $\alpha=-1, \beta=-2$, and break energy $E_{o}=150$

1) These values are based on fluxes corrected for a scale error, see footnote in [9]. I thank J. Brainerd for supplying the BATSE detector response and atmospheric scattering matrices. 

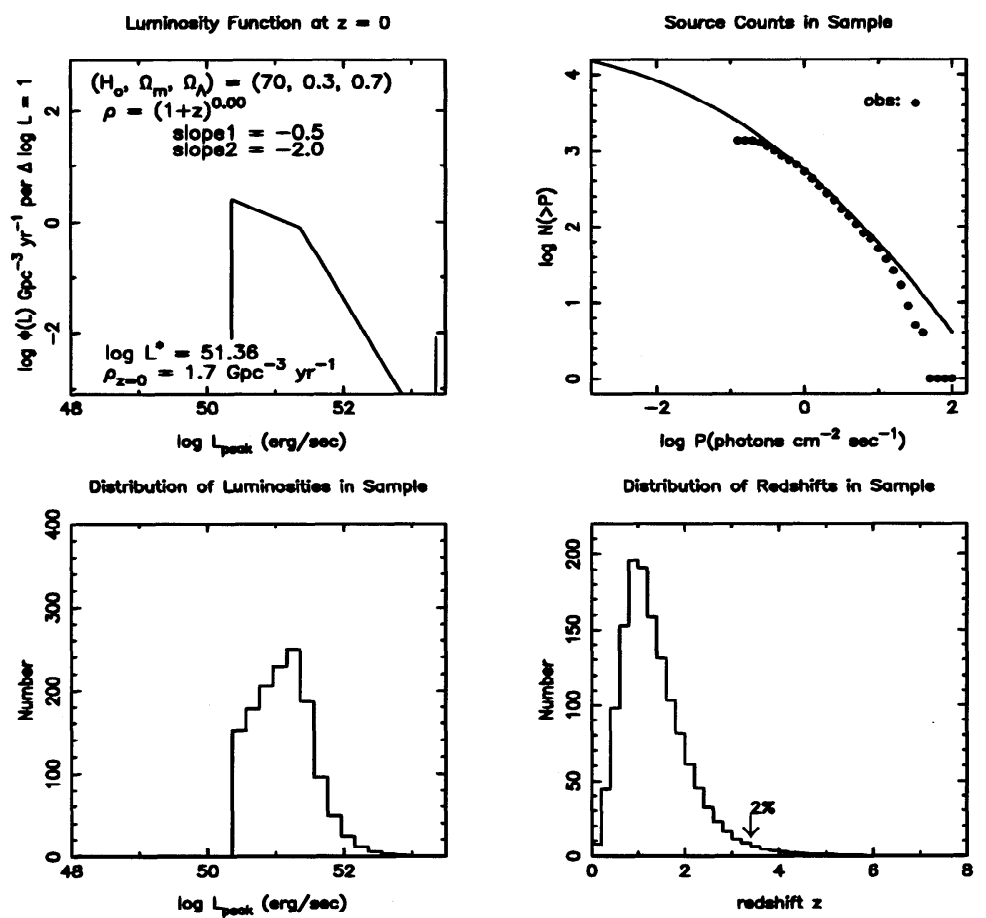

FIGURE 2. Predictions based on a GRB luminosity function with no density evolution

$\mathrm{keV}$. In Figure 1, we show the results for a typical model of the luminosity function, extending from $L^{*} / 10$ to $100 L^{*}$. The density evolution $\rho=(1+z)^{3.32}$ amounts to a factor of 10 at $z=1$ and is then constant at $z>1$. The local density is $30 \%$ lower than that for an open universe with $q_{o}=0.1$ [9]. As shown in Figure 1, the predicted $N(>P)$ distribution agrees well with the observations. The largest GRB redshift so far observed is 3.42 for GRB 971214 [7]. Based on the expected redshift distribution, the probability of finding a redshift of $z=3.4$ or larger in the BD2 sample is $6 \%$. The median redshift is 1.5 and the largest redshift among the 1391 GRB in the BD2 sample is $z=9.3$ according to this model.

In Figure 2, we show the results if there is no densily evolution. Compared to the previous case, $L^{*}$ is down by a factor of 2 and the local space density up by a factor of 15 . The predicted source counts fall below the observed points. The redshift distribution has only $2 \%$ of GRBs at a redshift of 3.4 or above.

\section{THE SUPERNOVA CONNECTION}

If the light curves of GRB afterglows show evidence for a supernova of Type Ib/c [4], the question arises whether all such supernovae could be associated with GRBs. 

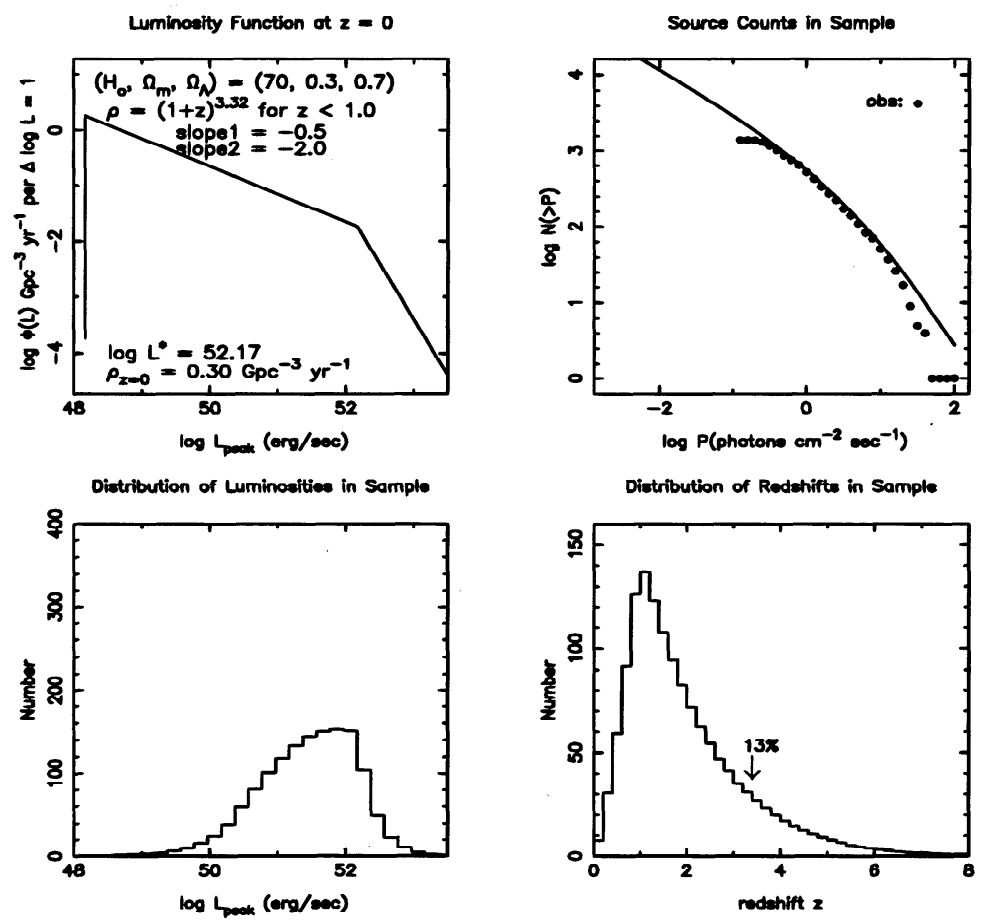

FIGURE 3. Predictions based on a luminosity function extending from $L^{*} / 10000$ to $100 L^{*}$

In the following, we update a discussion of this issue by Lamb [8]. If the gamma-ray emission is beamed over a fraction $f_{\text {beam }}$ of the celestial sphere, the local GRB rate is roughly (0.1-3) $f_{\text {beam }}^{-1} \mathrm{Gpc}^{-3} \mathrm{yr}^{-1}$, where the range mostly reflects the effect of density evolution (up to a factor of 10 at $z=1$ ), or no density evolution [9]. The rate of Type $\mathrm{Ib} / \mathrm{c}$ supernovae [5] in spiral galaxies is $\sim 10^{-13} \mathrm{~L}_{\odot}^{-1} \mathrm{yr}^{-1}$. With a luminosity density of $\sim 10^{8} \mathrm{~L}_{\odot} \mathrm{Mpc}^{-3}$ [3] for spirals, this corresponds to $\sim 10^{4} \mathrm{SN}$ $\mathrm{Gpc}^{-3} \mathrm{yr}^{-1}$. If $f_{\text {beam }}=10^{-2}$, then only 1 in (1000-30) SN Type Ib/c could have an associated GRB. If every Type Ib/c supernova harbors a GRB, $f_{\text {beam }}$ would have to be as small as $10^{-5}-10^{-3.5}$.

If GRB 980425 is associated with the SN 1998bw, its peak luminosity is $\log L=$ 46.7 [6]. We explored a luminosity function model that extends a factor of $10^{4}$ below $L^{*}$ (Fig. 3). Even though the luminosity function reaches down to around $\log L \sim 48$, the distribution of observed luminosities predicts only one GRB to have $\log L<48.5$, or a probability of around $0.1 \%$. Given this low probability, the identification of GRB 980425 with SN 1998bw can only be understood if it represents a separate population of low-luminosity bursts. Assuming that $30 \%$ of the observed GRBs are of low luminosity, we show in Figure 4 the expected distributions for the remaining GRBs assuming that they have a luminosity function 

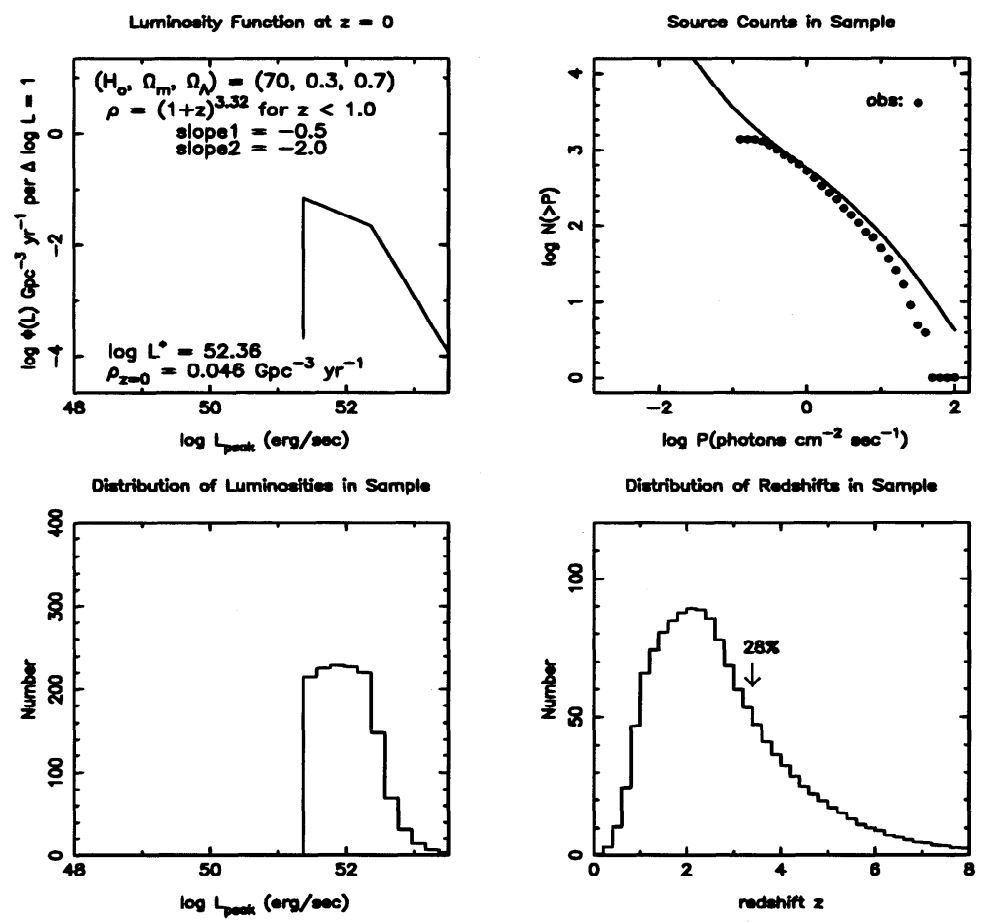

FIGURE 4. Assuming that $30 \%$ of observed GRB are of low luminosity, we show predicted distributions for the remaining GRBs for a given model of the luminosity function.

identical in shape to that of Figure 1. The fit of $N(>P)$ to the obscrved distribution is poor, indicating that at most $30 \%$ of observed GRBs can have a low luminosity.

\section{REFERENCES}

1. Bahcall, N. A., Ostriker, J. P., Perlmutter, S., and Steinhardt, P. J., Science 284, 1481 (1999).

2. Band, D. L., et al., ApJ 413, 281 (1993).

3. Binggeli, B., Sandage, A. and Tammann, G. A., in Ann. Rev. Astron. Astroph. 26, 509 (1988).

4. Bloom, J. S., et al., Nature 401, 453 (1999).

5. Cappellaro, E., et al., $A \& A$ 322, 431 (1997).

6. Galama, T. J., et al., A $6 A$ Suppl. 138, 465 (1999).

7. Kulkarni, S. et al., Nature 393, 35 (1998).

8. Lamb, D. Q., A\&A Suppl. 138, 607 (1999).

9. Schmidt, M., ApJ 523, L117 (1999).

10. Schmidt, M., A 8 A Suppl. 138, 409 (1999). 